\title{
Modeling of Digital Ecosystems: Challenges and Opportunities
}

\author{
John Krogstie \\ IDI, NTNU, Trondheim, Norway \\ krogstie@idi.ntnu.no
}

\begin{abstract}
Digital ecosystems' is a metaphor inspired by natural ecosystems which describes a set of distributed, adaptive, and open socio-technical systems. Being parts of such ecosystems, individual persons, public and private organisations are becoming increasingly dependent on each other. When such cooperation moves beyond simple buying and selling of goods and well-defined services, there is a need for a flexible infrastructure that supports not only information exchange, but also collaborative knowledge creation, evolution and sharing across a number of cooperation and collaborative networks that traditionally work in a bottom-up and rather improvised way. We will in this paper look at how techniques and approaches to modelling used e.g. for enterprise architecture and collaborative networks should evolve to support the development, support and evolution of digital ecosystems.
\end{abstract}

\section{Introduction}

All organizations are dependent on an application systems portfolio supporting its current and future tasks, and newcomers in any area are dependent on establishing a similar application portfolio quickly in a way that can evolve with changed business needs, technological affordances and expectations among co-operators, competitors and customers. An increasing fraction of the value creation in modern society comes from knowledge work using ICT. Such knowledge work is vital to meeting the grand challenges of today. As stated in the Digital Agenda for Europe [1], "Smart use of technology and exploitation of information will help us to address the challenges facing society like supporting an ageing society, climate change, reducing energy consumption, improving transportation efficiency and mobility, empowering patients and ensuring the inclusion of persons with disabilities".

The current organization of knowledge work result in a waste of ideas, knowledge, and solutions, which are not put into use where they are developed, and not exploited by others. An approach to address this is using 'open innovation' [2]. Open innovation will have to rely heavily on ICT, facilitating virtual communities of nomadic, human/ organizational actors, co-working on partially shared digital artefacts [3]. The term digital ecosystem has recently been used to generalize such communities, with focus on that their actors constantly interact and cooperate with other actors in both local and remote ecosystems. Examples of digital ecosystems are communities for Creative 
Commons and Open Source (OSS), social media networks as in Facebook, blogs and around computer games, virtual organizations, or voluntary groups of citizen. Note that we use the term in a wider context than what is termed business ecosystem in the collaborative networks literature [4] (a cluster or industry district).

A number of needs can be identified for supporting digital ecosystems. We will in this article discuss these issues highlighting the application and possible changed role of modelling techniques. In section 2 we describe the role of modelling in information systems development in general. In section 3 some traits of digital ecosystems are described, and section 4 describes a vision of the role of modelling in this landscape.

\section{The Role of Modeling and Quality of Models}

Information system modelling in general and modelling of collaborative networked organizations [5] is usually done in some organizational setting. One can look upon organizations and their information systems abstractly to be in a certain state (the current state, often represented as a descriptive 'as-is' model) that are to be evolved to some future wanted state (often represented as a prescriptive 'to be' model). These states are often modelled, and the state of the organization is perceived (differently) by different persons through these models. This open up for different usage areas of conceptual models as described e.g. in [6, 7].

1. Human sense-making: The model of the current state can be useful for people to make sense of and learn about the current situation as it is perceived.

2. Communication between people [8].

3. Computer-assisted analysis: To gain knowledge about the situation through simulation or deduction, often by comparing a model of the current state and a model of a future, potentially improved state.

4. Model deployment and activation: To integrate the model of the future state in an information system directly. Models can be activated in three ways:

a. Through people, where the system offers no active support.

b. Automatically, where the system plays an active role, as in an automated workflow system.

c. Interactively, the computer and the users co-operate on the process [9].

5. To give the context for a traditional system development project, without being directly activated.

6. Achieve acceptance of solution due to acting as a common ground

7. Quality assurance, ensuring that e.g. an organization acts according to a certified process achieved for instance through an ISO-certification process.

SEQUAL (Semiotic Quality Framework) is a generic framework for assessing quality of models $[10,11]$. The framework has earlier been used for evaluation of modelling and modelling languages of a large number of perspectives, including data, object, process, enterprise, and goal-oriented modelling. Quality has been defined referring to the correspondence between statements belonging to the following sets: 
- $G$, the goals of modelling.

- $\boldsymbol{L}$, the language extension, i.e., the set of all statements that are possible to make according to the rules of the modelling languages used.

- $\boldsymbol{D}$, the domain, i.e., the set of all statements that can be stated about the situation.

- $\boldsymbol{M}$, the externalized model itself.

- $\boldsymbol{K}$, the explicit knowledge relevant to the domain of the audience.

- $\boldsymbol{I}$, the social actor interpretation, i.e., the set of all statements that the audience interprets that an externalized model consists of.

- $\boldsymbol{T}$, the technical actor interpretation, i.e., the model as 'interpreted' by tools.

The main quality types are:

- The deontic quality of a model relates to that all statements in the model $\boldsymbol{M}$ contribute to fulfilling one or more of the goals of modelling $\boldsymbol{G}$, and that all the goals of modelling $\boldsymbol{G}$ are addressed through the model $\boldsymbol{M}$. In particular, one include under deontic quality the extent that the participants after interpreting the model learn based on the model (increase $\boldsymbol{K}$ ) and that the audience are able to change the domain $\boldsymbol{D}$ if this is beneficially to achieve the goals of modelling.

- The goal defined for social quality is agreement among social actor's interpretations.

- Perceived semantic quality is the similar correspondence between the social actor interpretation $\boldsymbol{I}$ of a model $\boldsymbol{M}$ and his or hers current knowledge $\boldsymbol{K}$ of domain $\boldsymbol{D}$.

- Pragmatic quality is the correspondence between the model $\boldsymbol{M}$ and the actor interpretation $(\boldsymbol{I}$ and $\boldsymbol{T})$. One differentiates between social pragmatic quality (to what extent people understand the models) and technical pragmatic quality (to what extent tools can be made that can interpret the models).

- Semantic quality is the correspondence between the model $\boldsymbol{M}$ and the domain $\boldsymbol{D}$. This includes validity and completeness.

- Syntactic quality is the correspondence between the model $\boldsymbol{M}$ and $\boldsymbol{L}$.

- Empirical quality deals with comprehensibility and predictable error frequencies when a model $\boldsymbol{M}$ is read or written by different social actors.

- Physical quality: The main goal is that the externalized model $\boldsymbol{M}$ is physically available to the relevant social and technical actors for interpretation $(\boldsymbol{I}$ and $\boldsymbol{T})$.

\section{Characteristics of Digital Ecosystems}

The long-term trend in ICT has been towards IT-systems being developed and evolved further and further away from the users of the system [12]. We see a development in the direction of systems to a larger degree being supported by virtual communities of nomadic, human/organizational actors, co-working on partially shared digital artefacts [13]. The term 'digital ecosystem' has recently been used to generalize such communities. Such systems are characterized by self-organization, scalability and sustainability, providing both economic and social value. Digital ecosystems are part of an even larger area called digital ecologies [14]. 
However, the existing digital ecosystems have limited scope, various degree of transparency, insufficient support for search and evaluation of useful quality artefacts, and none does fully support a wide range of shared artefacts from a wide range of actors. There are two main variants of digital ecosystems; content ecosystem and software ecosystems.

Content ecosystems are networks that deal with creation and sharing of artistic and intellectual artefacts. ICT have increasing impact on participative and democratic processes, and this impact will continue to grow with the increasing personalization, witnessed through the increase of social networking and user generated content and services. Internet already allows highly visual and multimodal interactions, and these interactions will become represented through richer means.

Software ecosystems are "a set of businesses functioning as a unit and interacting with a shared market for software and services, together with relationships among them. These relationships are frequently underpinned by a common technological platform and operate through the exchange of information, resources, and artefacts" [15]. See also work on software ecosystems for product families [13], more general software systems [16], and guidelines for using such ecosystems [15]. For instance within open source systems (OSS) a large number of co-evolved software components are freely available. The quality is variable and often poorly documented. Yet, many organizations now integrate OSS components into their own applications, and some also contribute back [17]. Traditional customers - like municipalities - cooperate to provide improved e-services for their inhabitants. And end-users - even kids - are becoming their own developers.

To address combined digital content and software ecosystems, there must be substantial and concerted improvements of the state-of-the-art in three traditionally unrelated and partially isolated research areas. Enterprise architecture and enterprise modelling, new business models and data management.

\section{$4 \quad$ Modeling of Digital Ecosystems}

The kind of modelling we are looking on in our work in particular applies to the first two areas above, i.e. enterprise and business modelling. Organizations are becoming less self-sufficient and increasingly dependent on partners and other actors, e.g. by outsourcing non-core activities. However, when such cooperation moves beyond simple buying and selling of goods and well-defined services, there is a need for a flexible infrastructure that supports both information exchange, knowledge creation, evolution and sharing across the different collaborative networks that tend to work in a bottom-up and rather improvised way. Within many organizations, it has become customary to develop enterprise architectures [18]. Ecosystem architecture takes the ideas of enterprise architecture to a higher level of abstraction, looking upon the support of a more fluid landscape of business actors providing and consuming services for information systems support in an organizational setting. In this way it extends the process perspective in BPM-in-the-Large [19] to a wider setting. In also extend work in collaborative networks, such as ARCON [5]. ARCON offers an 
approach to model collaborative networks $(\mathrm{CN})$, including: the $\mathrm{CN}$ life cycle dimension, the $\mathrm{CN}$ environmental perspectives dimension, and the $\mathrm{CN}$ modelling intent dimension.

A new approach to enterprise integration is needed. User-initiated software applications and enterprise mash-ups should be based on active knowledge modelling (AKM) and supports learning [20]. For this to function, one must support more open business models. In this, one must consider financial success, sustainability, competition, copyright and licensing, and the impact on work processes, leadership, internal coordination, work processes, strategy and planning. The open innovation approach [2] is often chosen as a basic cooperation mechanism. Companies should allow freer ("open") import and export of ideas and knowledge concerning products, processes and business models that flow between organization and their environments. Indeed, more openness will provide a larger set of possible business opportunities. Problems connected to IP and revenue sharing must be considered. Furthermore, an open innovation strategy must be reflected not only in the business models, but also by revised behaviour (process practice) and in new thinking patterns. The SEM modelling language [15] attempts to analyze the business along customersupplier lines. Furthermore, the $E^{3}$ value model [21] describes value-generation and value-exchange among partners in a value network. Other relevant approaches are BMO [22] and ARCON [5].

There are two main scenarios for the future use of modelling in this setting. What we term the steady state scenario, where modelling continues to be a somewhat esoteric activity for a limited number of experts is of course one possibility. The more optimistic scenario in our view is that abstraction techniques such as modelling are taken into use in an increasing number of areas, to make it possible to at all be able to manage this development. One striking aspect is that the number and variety of stakeholders that will need to relate to models of some sort will increase. Given the increased educational level in most countries, it is not unlikely that also more people will be able to relate to these types of abstractions.

Using the sets in SEQUAL, we predict the following under this scenario (in Fig. 1, the areas we foresee large changes are shown with stippled lines):

- G: The same list of goals and applications of modelling that is described in Section 2 will still be relevant, but emphasis on less formal, interactive approaches will increase to be able to support the more federated landscape needed to address digital ecosystems.

- D: The range of relevant domains is increasing given that systems to an increasing degree ranges across and is expected to integrate a number of areas. Business aspects must to a larger degree be integrated with more traditional enterprise aspects such as goals, processes, products, systems and data.

- $\mathrm{K}$ : One need to deal with a more varied set of stakeholders, with a more varied set of skills and knowledge. Not only do you need to align IT-experts with business-experts, but also people across a large range of expertise, and across organizations, being used to express their knowledge in different notations.

- L: Using domain specific modelling, the possibilities of tailoring the language to fit the domain, and the knowledge of the stakeholders have increased. To bring 
more people into (semi-) formal modelling these possibilities will have to be exploited to a larger degree. Thus rather than having a consolidation of modelling languages like the one done in object-oriented design with UML, there will be an increasing number of variants of modelling languages. We will also see a mix of richer media components being integrated with the more traditional "box and arrow"-conceptual modelling notations, thus supporting also richer meta-meta models defining and limiting the type of constructs to include in models.

- $\quad \mathrm{T}$ : An increasing number of tools will be available to extract model information from raw data, e.g. in the area of process mining and semantic web. In addition, tools for meta-meta modelling and meta-modelling will be more common.

- M: Models will be pervasively available being coordinated in a federated manner. Models will be across meta-levels in an increasing degree (compared to the models in traditional software engineering being primarily on the type level). Models, in particular interactive models [9] will have a larger value in themselves, acting to a larger extend as knowledge commons and open models (http://www.openmodels.at/).

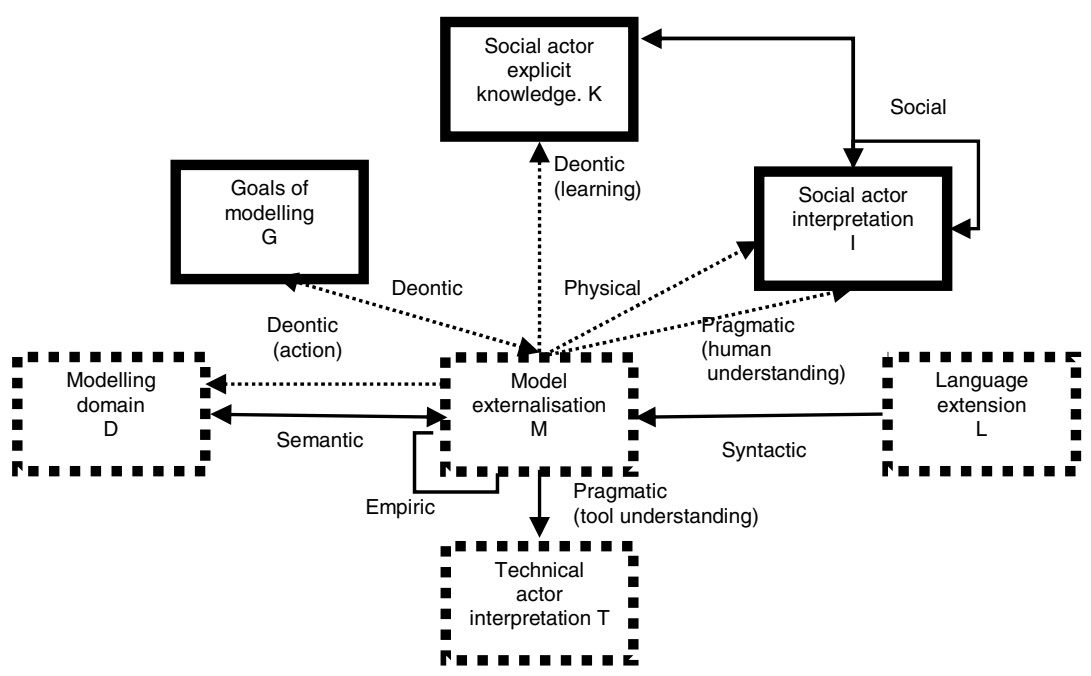

Fig. 1. SEQUAL with areas that is changing when modelling digital ecosystems

We believe the core dimensions of SEQUAL will be relevant for discussing also models used in digital ecosystems. On the other hand, a number of specializations might be envisaged. We will briefly discuss some main aspects here.

- Deontic quality: Models will be more important, due to increased dependencies across traditional organizational boundaries and needs to be handled in a more professional manner [8].

- Social quality: This will be important in smaller communities, and in interfaces between communities, but less needed across federation. Note on the other hand 
that since different stakeholder groups might see different views of the overall model, possible visualized in radically different ways, the effort to assure that they comprehend the models equally will potentially have to increase [23].

- Pragmatic quality: Given that more types of stakeholders are involved, this is of increasing importance. Different techniques can be used for different types of stakeholder, supporting multiple views for different stakeholder types on the same model to ensure individual comprehension.

- Semantic quality: The federated approach needed for modelling will bring new challenges as for how we look upon the semantic quality of the overall model. Whereas semantic quality in smaller domains would be followed up much as before (i.e. looking at the feasible (perceived) completeness and validity), one would to a larger degree need to be able to live with inconsistencies across federations. In connection to this, it would be important to be able to identify those aspects of the models across domains that need to be consistent.

- Syntactic quality: Syntactic quality can be looked upon as trivial in a sense, since adherence can be enforced. On the other hand, one often sees that one extend languages with new aspects in an (not always conscious) attempt to turn semantic problems into syntactic problems. New tools based on meta-modelling makes this easier to do, and then makes in even more important to do right in the sense that one do not end up with too restricted languages.

- Empirical quality: Support for empirical quality will be more built in, e.g. in tools that build up models from raw data in process mining, thus integrating information visualization tools and modelling tool. Different meta-meta models can necessitate rethinking guidelines for achieving empirical quality [6].

- Physical quality: Rather than being based on central repositories, more distributed, federated storage of model fragments must be available, utilizing standard interchange formats and supporting model mash-ups. What part of the total model that should be available for each partner must be addressed.

\section{Concluding Remarks}

From the above descriptions, we see that the technical challenges and opportunities with digital ecosystems give new challenges and opportunities for model-based techniques. In a way many of the core problems are not new. Even if the use of modelling needs to be extended and improved, general categories underlying discussions on quality of models as described in [10] remains relevant, although need to be adapted to e.g. quality of interactive models $[9,20,24]$. We plan to pursue this work by working on case studies in selected domains including public sector, smartgrids and the petroleum industry to investigate more concretely how to extend the techniques described in section 4 for modelling of digital ecosystems.

Acknowledgements. This work is part of ongoing cross-disciplinary work on this area. I would in particular like to thank my colleagues Letizia Jaccheri, Jon Atle Gulla, Reidar Conradi, Tonje Osmundsen, Per Morten Schiefloe, Guttorm Sindre, Kjetil Nørvaag, Martha Molinas, and Alf Inge Wang in this regard. 


\section{References}

1. European Commission, A Digital Agenda for Europe, COM, Brussels (2010)

2. Chesbrough, H.: Open Services Innovation. Rethinking your Business to Growth and Compete in a New Era. Jossey-Bass, San Francisco (2011)

3. Ali Babar, M., Dingsøyr, T., Lago, P., van Vliet, H. (eds.): Software Architecture Knowledge Management - Theory and Practice. Springer (2009)

4. Camarinha-Matos, L.M.: Taxonomy of Collaborative Networks Forms, FInES (2012)

5. Afsarmanesh, H., Camarinha-Matos, L.M.: The ARCON modeling framework. Springer, New York (2008)

6. Nossum, A., Krogstie, J.: Integrated Quality of Models and Quality of Maps. In: Halpin, T., Krogstie, J., Nurcan, S., Proper, E., Schmidt, R., Soffer, P., Ukor, R. (eds.) Enterprise, Business-Process and Information Systems Modeling. LNBIP, vol. 29, pp. 264-276. Springer, Heidelberg (2009)

7. Krogstie, J.: Model-Based Development and Evolution of Information Systems: A Quality Approach. Springer, London (2012)

8. Wesenberg, H.: Enterprise Modeling in an Agile World. In: Johannesson, P., Krogstie, J., Opdahl, A.L. (eds.) PoEM 2011. LNBIP, vol. 92, pp. 126-130. Springer, Heidelberg (2011)

9. Krogstie, J., Jørgensen, H.D.: Quality of Interactive Models. Paper Presented at the First International Workshop on Conceptual Modelling Quality, IWCMQ 2002 (2002)

10. Krogstie, J., Sindre, G., Jørgensen, H.D.: Process Models as Knowledge for Action: A Revised Quality Framework. EJIS 15(1), 91-102 (2006)

11. Krogstie, J., Sølvberg, A.: Information systems engineering - Conceptual modeling in a quality perspective. Kompendiumforlaget, Trondheim (2003)

12. Davidsen, M.K., Krogstie, J.: A longitudinal study of development and maintenance. Information and Software Technology 52, 707-719 (2010)

13. Bosch, J.: Architecture Challenges for Software Ecosystems. In: Proc. Fourth European Conference on Software Architecture, ECSA - Companion Volume, Copenhagen, Denmark, August 23-26, pp. 93-95. ACM Press (2010)

14. Vyatkin, V., Zhabelova, G., Ulieru, M., McComas, D.: Toward Digital Ecologies: Intelligent Agent Networks Controlling Interdependent Infrastructure. In: IEEE Conference on Smart Grid Communications, Gaithersburg (October 2010)

15. Jansen, S., Finkelstein, A., Brinkkemper, S.: A sense of community: A research agenda for software ecosystems. In: Proc. 31st International Conference on Software Engineering, ICSE, Vancouver, Canada, May 16-24, pp. 187-190 (2009)

16. Hunink, I., van Erk, R., Jansen, S., Brinkkemper, S.: Industry taxonomy engineering: the case of the European software ecosystem. Proc. In: Fourth European ECSA - Companion Volume, Copenhagen, Denmark, Aug. 23-26, pp. 111-118. ACM Press (2010)

17. Hauge, Ø., Ayala, C., Conradi, R.: Adoption of Open Source Software in SoftwareIntensive Industry - A Systematic Literature Review. Information and Software Technology 52(11), 1133-1154 (2010)

18. Lankhorst, M.: Enterprise Architecture at Work. Springer (2009)

19. Houy, C., Fettke, P., Loos, P., van der Aalst, W.M.P., Krogstie, J.: BPM-in-the-Large Towards a Higher Level of Abstraction in Business Process Management. In: Janssen, M., Lamersdorf, W., Pries-Heje, J., Rosemann, M. (eds.) EGES 2010 and GISP 2010. IFIP Advances in Information and Communication Technology, vol. 334, pp. 233-244. Springer, Heidelberg (2010)

20. Lillehagen, F., Krogstie, J.: Active Knowledge Modeling of Enterprises. Springer (2008) 
21. Gordijn, J., Yu, E., van der Raadt, B.: E-service design using $i^{*}$ and $\mathrm{e}^{3}$ value modeling. IEEE Software 23(3) (2006)

22. Osterwalder, A., Pigneur, Y., Smith, A.: Business Model Generation. Wiley (2010)

23. Krogstie, J., Dalberg, V., Jensen, S.M.: Process modeling value framework. In: Manolopoulos, Y., Filipe, J., Constantopoulos, P., Cordeiro, J. (eds.) ICEIS 2006. LNBIP, vol. 3, pp. 309-321. Springer, Berlin (2008)

24. Krogstie, J., Jørgensen, H.D.: Interactive Models for Supporting Networked Organisations. In: Persson, A., Stirna, J. (eds.) CAiSE 2004. LNCS, vol. 3084, pp. 550-563. Springer, Heidelberg (2004) 